\title{
Rectal neuroendocrine tumor developing lateral lymph node metastasis after curative resection: a case report
}

\author{
Yoshihisa Tokumaru, Nobuhisa Matsuhashi*, Takao Takahashi, Hisashi Imai, Yoshihiro Tanaka, Naoki Okumura, \\ Kazuya Yamaguchi and Kazuhiro Yoshida
}

\begin{abstract}
Background: Among gastrointestinal neuroendocrine tumors (NETs), rectal NETs account for about one-third of all tumors. Despite the occasional observation of lateral lymph node metastasis in patients with rectal NETs, lateral lymph node recurrence is rare. We present a rare case of lateral lymph node recurrence after curative resection of a rectal NET.

Case presentation: A 55-year-old man presented with fecal occult blood and colonoscopy revealed a mass in the distal rectum. Systematic computed tomography scan showed no evidence of regional lymph node or distant metastasis. The patient underwent laparoscopic intersphincteric resection and D2 lymph node dissection with diverting stoma. Diverting stoma closure was performed 6 months after the initial operation. Pathological diagnosis was NET of the rectum, grade 2, T1 b, NO, Stage I without lymphovascular invasion. At 54 months after the surgery, recurrence in a left lateral lymph node was suspected and lymph node dissection was performed. The pathological diagnosis of the specimen was consistent with lateral lymph node metastasis of a recurrent rectal NET. To our best knowledge, there are no case reports in English of lateral lymph node recurrence after curative resection of a rectal NET, grade 2, T1b, NO, Stage I without lymphovascular invasion.

Conclusion: Considering that patients with lateral lymph node metastasis have worse survival than those without metastasis in rectal cancer, if complete resection of the tumor can be achieved for lateral lymph node recurrence, surgery may be an important option in the strategy to treat this condition.
\end{abstract}

Keywords: Neuroendocrine tumor, Lateral lymph node metastasis, Rectum, Case report

\section{Background}

Neuroendocrine tumors (NETs), which arise from neuroendocrine cells, are rare, and rectal NETs account for about one-third of all gastrointestinal NETs [1-3]. The incidence of rectal NETs is increasing with the development of better diagnostic tools and improved knowledge [4]. In general, rectal NETs tend to show characteristics of a less aggressive tumor compared with NETs of the

\footnotetext{
* Correspondence: nobuhisa517@hotmail.com

Department of Surgical Oncology, Gifu University School of Medicine, 1-1 Yanagido, Gifu, Gifu 501-1194, Japan
}

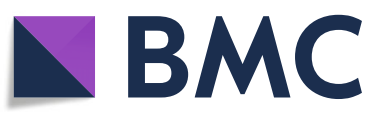

(c) The Author(s). 2020 Open Access This article is licensed under a Creative Commons Attribution 4.0 International License, which permits use, sharing, adaptation, distribution and reproduction in any medium or format, as long as you give appropriate credit to the original author(s) and the source, provide a link to the Creative Commons licence, and indicate if changes were made. The images or other third party material in this article are included in the article's Creative Commons licence, unless indicated otherwise in a credit line to the material. If material is not included in the article's Creative Commons licence and your intended use is not permitted by statutory regulation or exceeds the permitted use, you will need to obtain permission directly from the copyright holder. To view a copy of this licence, visit http://creativecommons.org/licenses/by/4.0/. The Creative Commons Public Domain Dedication waiver (http://creativecommons.org/publicdomain/zero/1.0/) applies to the data made available in this article, unless otherwise stated in a credit line to the data. consideration of the risk factor of lymph node or distant metastasis is important. Fahy et al. stratified patients at risk by using parameters such as tumor size, mitotic rate, lymphovascular invasion, and depth of invasion of the obtained tissue specimen [5]. From previous reports, $5.9-6.5 \%$ of patients with rectal NETs were confirmed to have lateral lymph node metastasis after rectal resection $[6,7]$. However, the recurrence of lateral lymph node metastasis is very rare, and to the best of our knowledge, there are no case reports in English of lateral lymph 
node recurrence after curative resection of a rectal NET. Here, we present a rare case of lateral lymph node recurrence after curative resection of a rectal NET, grade 2, T1b, N0, Stage I without lymphovascular invasion.

\section{Case presentation}

A 55-year-old man was admitted to a provincial hospital with fecal occult blood. Colonoscopy revealed a submucosal tumor with depression in the anterior wall of the distal rectum (Fig. 1). The tumor was diagnosed as a rectal NET following pathological examination of the biopsy specimen, and he was referred to our hospital for further examination. Endorectal endoscopic ultrasound revealed a 14-mm oval tumor with deep invasion to the submucosa layer. The tumor was located at $1.8 \mathrm{~cm}$ from the anal verge. Systematic computed tomography (CT) revealed no evidence of regional lymph node metastasis or distant metastasis such as that to the liver or lung. On the basis of these findings, we performed laparoscopic subtotal intersphincteric resection and D2 lymph node dissection with diverting stoma. The macroscopic finding of the resected specimen revealed an oval-shaped tumor with depression. Pathological examination with hematoxylin and eosin (HE) staining showed the tumor cells spreading in a rosette-like pattern. Immunohistochemical staining revealed the tumor cells to be positive for chromogranin A and synaptophysin, with a Ki-67 labeling index of 3\% (Fig. 2). Pathological diagnosis was NET of the rectum, G2, T1b (invasion to submucosa), N0, Stage I without lymphovascular invasion. Diverting stoma closure was performed 6 months after the initial operation. A follow-up abdominopelvic CT scan at 12 months after surgery detected a 4-mm mass in the left

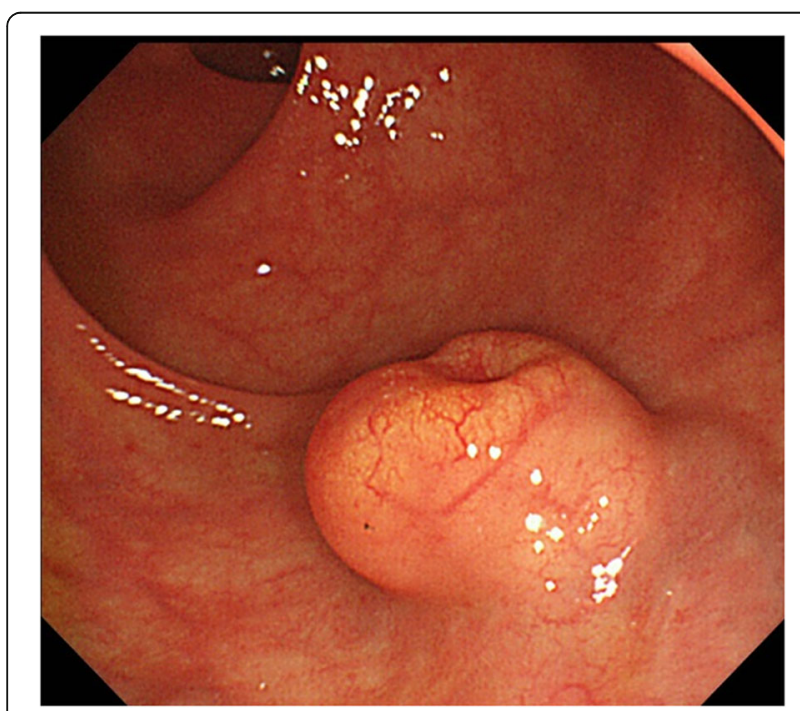

Fig. 1 Colonoscopy of the primary site shows a 14-mm submucosal tumor with central depression located at the anterior wall of the distal rectum internal iliac region (Fig. 3a). The mass was followed with abdominopelvic CT every 6 months and occasional positron emission tomography (PET)-CT. At 54 months after surgery, the mass had enlarged to $20 \mathrm{~mm}$ (Fig. 3b), but PET-CT did not show abnormal uptake in the tumor or in other distant organs. Because the mass had enlarged over time, we suspected it to be a single lateral lymph node recurrence, and we performed left lateral lymph node dissection. The resected specimen was again an oval-shaped mass of $14 \mathrm{~mm}$ in size (Fig. 4). The finding from HE staining was similar to that in the specimen resected at the primary surgery (Fig. 5a, b). Immunohistochemical staining again revealed the same findings as in the previous resected specimen, i.e., cells positive for chromogranin A and synaptophysin positive, but now the Ki67 labeling index had increased slightly to 5\% (Fig. 5c, d). On the basis of these findings, the pathological diagnosis was lateral lymph node recurrence. The patient was followed up with CT every 6 months and colonoscopy annually. At 42 months after the second surgery, the patient has shown no evidence of recurrence.

\section{Discussion}

Rectal NETs are the most common type of gastrointestinal NETs in Asia, and they account for $60-89 \%$ of all gastrointestinal NETs in Japan [8]. Due to the increasing numbers of endoscopies and improved diagnostic instruments, the incidence of rectal NET is increasing [9].

When determining a treatment strategy, consideration of the risk of recurrence is very important when choosing the type of surgical procedures to perform, such as endoscopic or transanal excision and laparoscopic or transabdominal resection. The National Comprehensive Cancer Network (NCCN) guidelines recommend radical resection with LN dissection for rectal NETs $>2 \mathrm{~cm}$ in diameter [10]. In agreement with this notion, the North American Neuroendocrine Tumor Society also recommends radical resection with $\mathrm{LN}$ dissection for rectal NETs $>2 \mathrm{~cm}$ in size and for $1-$ to $2-\mathrm{cm}$ tumors with muscular invasion or positive nodes $[8,9,11]$. Also, the European Neuroendocrine Tumor Society (ENETS) Consensus Guidelines recommend radical surgical resection, such as total mesorectal excision, for a tumor $>20$ $\mathrm{mm}$ because there is high risk for metastasis [2, 8]. For tumors of $<1 \mathrm{~cm}$, the ENETS Guidelines recommend endoscopic or transanal excision as the risk of metastasis is low $(<3 \%)[8,12]$. For tumor of 11 to $19 \mathrm{~mm}$ in size, despite the current notion of performing less invasive procedures, such as endoscopic excision, the optimal procedure for resection remains controversial. Also, it is debatable whether radical lymph node dissection should be performed for tumors of these sizes or if they should be treated with local excision only. Increasing evidence supports a minimally invasive treatment strategy of 


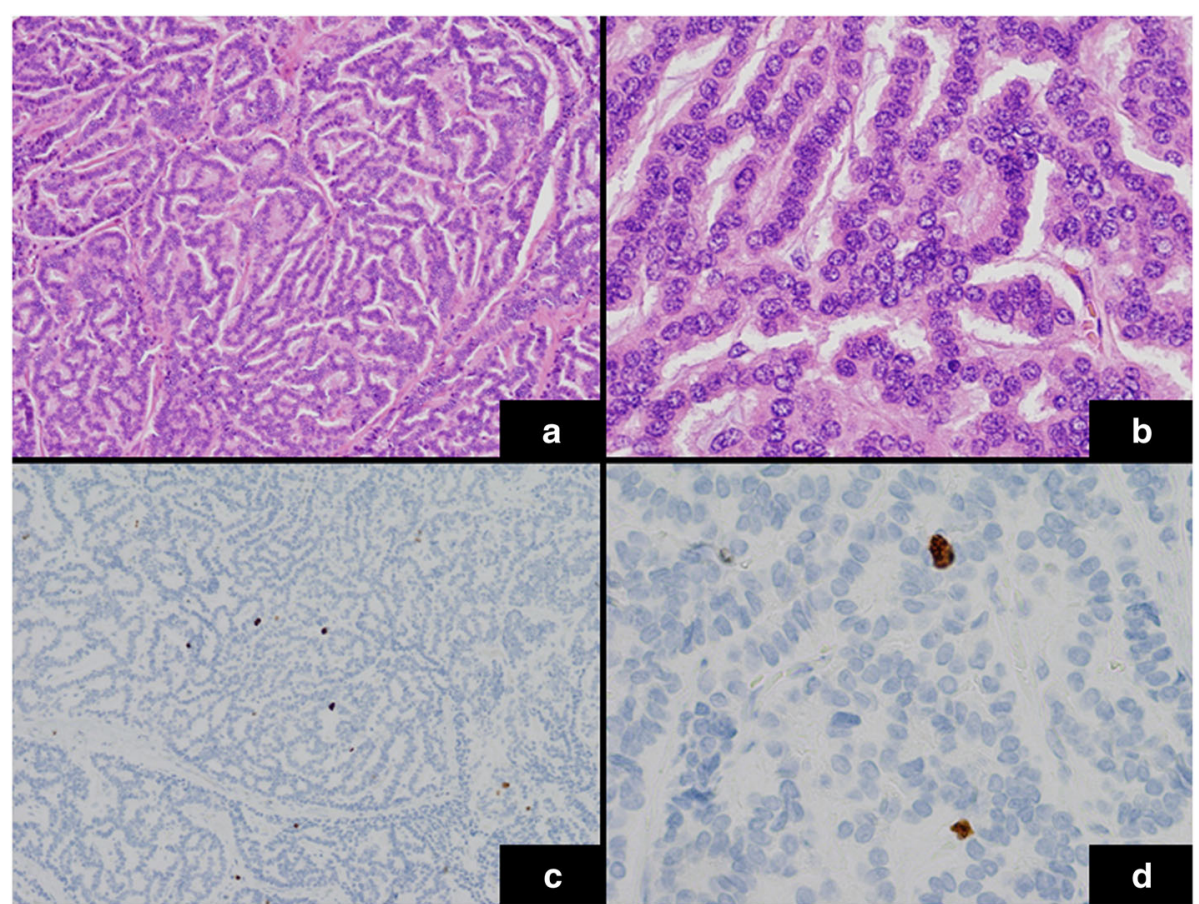

Fig. 2 Histopathological findings of the resected specimen (primary tumor). $\mathbf{a}, \mathbf{b}$ HE staining showed the tumor cells spreading in a rosette-like pattern. c, d Immunohistochemical staining for Ki-67 (labeling index $=3 \%$ ) (a, c original magnification $\times 100 ; \mathbf{b}$, $\mathbf{d}$ original magnification $\times 400$ )

endoscopic resection or dissection [13]. However, 7$18.6 \%$ of lymph node metastases come from tumors < $10 \mathrm{~mm}$ in size $[7,14,15]$. Given that most of these tumors harbor lymphovascular invasion, Matsuhashi et al. emphasized the importance of evaluating lymphovascular invasion of the resected specimen [16]. In the present case, the original tumor was $14 \mathrm{~mm}$ in size and had a depression on its surface. Also, the endorectal endoscopic ultrasound findings revealed the suspicion of tumor invasion into the deep submucosa layer. We thus decided to perform laparoscopic intersphincteric resection for this patient as the primary surgery.

There is no consensus regarding a treatment strategy for the recurrence of gastrointestinal NETs. For metastatic disease, the NCCN guidelines state that if complete resection is possible, surgery for both the metastatic site and the primary site can be performed [10]. Also, the North American Neuroendocrine Tumor Society (NANETS) states that despite there are no data with colorectal NETs, cytoreductive surgery would be an

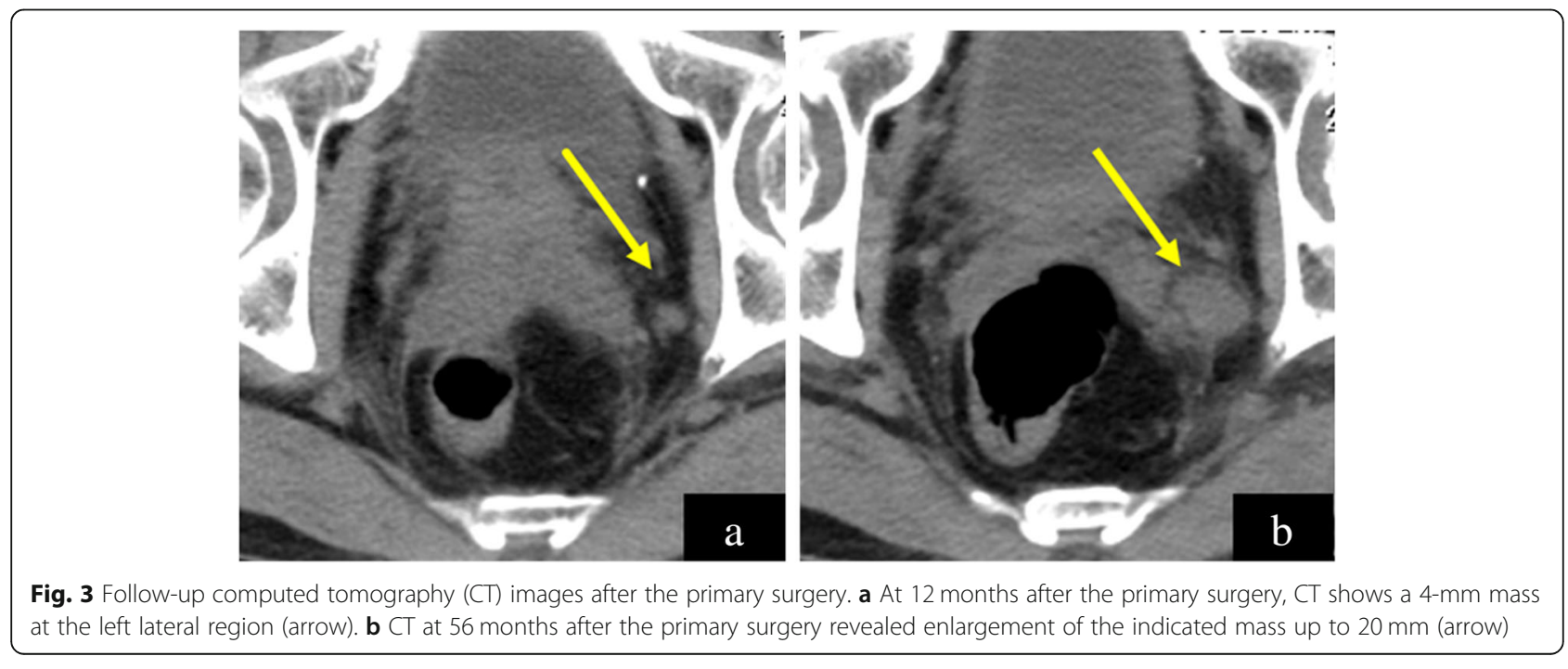




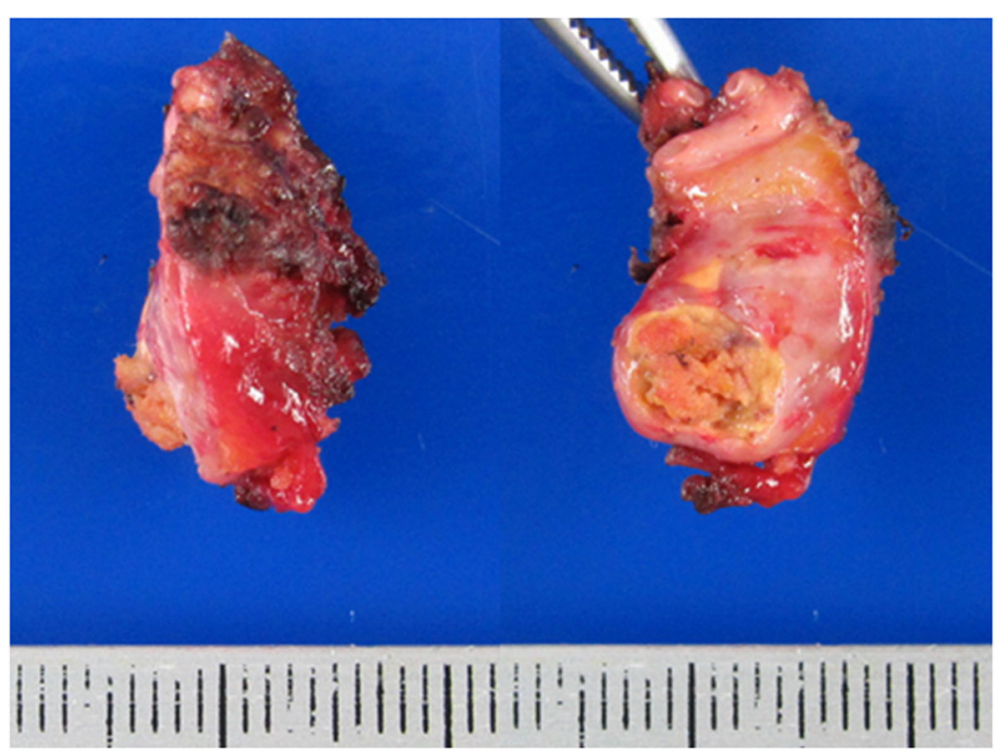

Fig. 4 The resected recurrence specimen was an oval-shaped 14-mm mass

option to perform if over $90 \%$ of the tumor is resected safely for other type of NETs. These statements imply that the lymph node dissection would be an option for the lateral lymph node recurrence like our case [11]. Glazer et al. reported the long-term survival of 172 patients with hepatic metastasis of NETs who underwent surgical procedures and found that their rate of 10-year overall survival was 50.4\% [17]. This result implies the possibility of a favorable long-term outcome for patients suffering the recurrence of a NET.

Regarding the surgical procedure of lateral lymph node dissection with rectal NET, there is no general

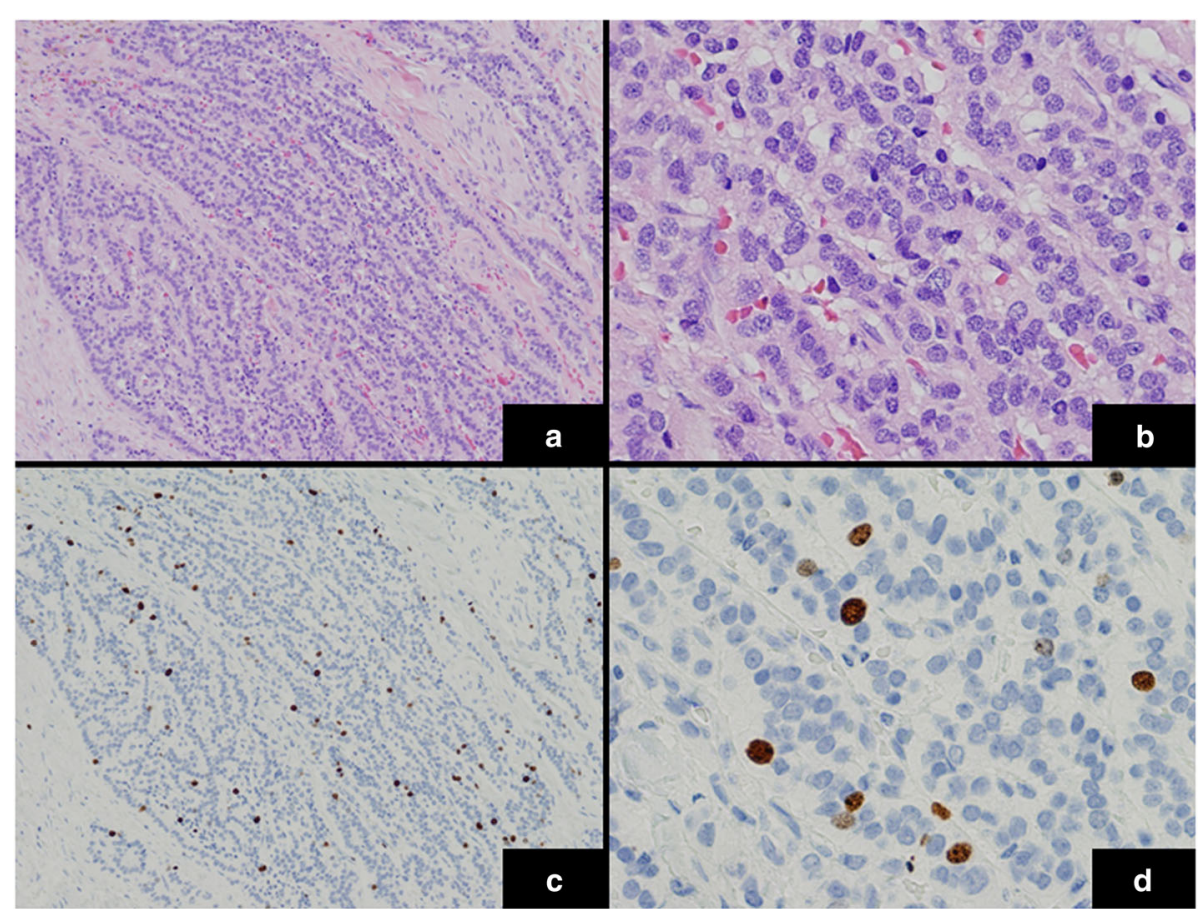

Fig. 5 Histopathological findings of the resected specimen (recurrent tumor). $\mathbf{a}, \mathbf{b} \mathrm{HE}$ staining of the recurrent tumor specimen revealed findings similar to those of the primary tumor. c, $\mathbf{d}$ Immunohistochemical staining for Ki-67 (labeling index $=5 \%$ ). (a, c original magnification $\times 100 ; \mathbf{b}$, $\mathbf{d}$ original magnification $\times 400$ ) 
agreement for its indication. In Japan, lateral lymph node dissection is recommended for rectal cancer with muscularis propria invasion that is located distal to the peritoneal reflection [18]. Takatsu et al. reported a single-center, retrospective study of the short- and longterm outcomes of curative surgery of 82 patients with rectal NET [7]. In their study, 5 of 6 patients who underwent lateral lymph node dissection had lymph node metastasis. In their report, the indication for performing lateral lymph node dissection was a patient who shows a lymph node of $>7 \mathrm{~mm}$. However, none of their $6 \mathrm{pa}-$ tients had local recurrence. Similar to the report of Takatsu et al., Ushigome et al. reported that they used a lymph node with $>7 \mathrm{~mm}$ in longitudinal diameter as the indication for the performance of lateral lymph node dissection [6]. In their study, they reported a positive rate of $86 \%$ after adopting this indication. Despite the small sample size, from these findings, lateral lymph dissection can be considered for cases of recurrence. Also, considering the fact that patients with lateral lymph node metastasis have worse survival than patients without metastasis in rectal cancer, if complete resection of the tumor can be achieved for lateral lymph node recurrence, surgery may be an important option in the treatment strategy.

\section{Conclusion}

We report a rare case of lateral lymph node recurrence after curative resection of a rectal NET, grade 2, T1b, N0, Stage I without lymphovascular invasion. Surgery may be an important option in the treatment strategy for lateral lymph node recurrence.

\section{Abbreviations}

NETs: Neuroendocrine tumors; CT: Computed tomography; PET-CT: Positron emission tomography-computed tomography; NCCN: National

Comprehensive Cancer Network

\section{Acknowledgements}

Not applicable

\section{Authors' contributions}

$Y T$ collected the patient's clinical data, and drafted the manuscript. TT helped in the management of the patient. HI, YT, NO, and KY collected the patient's clinical data. NM and KY made revision and supervision of the study. All authors read and approved the final manuscript.

\section{Funding}

None

Availability of data and materials

Not applicable

Ethics approval and consent to participate

Not applicable

\section{Consent for publication}

Not applicable

\section{Competing interests}

None of the authors have any conflicts of interest to declare
Received: 20 November 2019 Accepted: 19 March 2020

Published online: 13 April 2020

\section{References}

1. Dasari A, Shen C, Halperin D, Zhao B, Zhou S, Xu Y, et al. Trends in the incidence, prevalence, and survival outcomes in patients with neuroendocrine tumors in the United States. JAMA oncology. 2017;3(10): 1335-42.

2. Ramage JK, De Herder WW, Delle Fave G, Ferolla P, Ferone D, Ito T, et al. ENETS Consensus Guidelines update for colorectal neuroendocrine neoplasms. Neuroendocrinology. 2016;103(2):139-43.

3. Kitagawa Y, Ikebe D, Suzuki T, Hara T, Itami M, Yamaguchi T. Frequent presence of lymphovascular invasion in small rectal neuroendocrine tumors on immunohistochemical analysis. Digestion. 2017;95(1):16-21.

4. de Mestier L, Lorenzo D, Fine C, Cros J, Hentic O, Walter T, et al. Endoscopic, transanal, laparoscopic, and transabdominal management of rectal neuroendocrine tumors. Best Pract Res Clin Endocrinol Metab. 2019;101293.

5. Fahy BN, Tang LH, Klimstra D, Wong WD, Guillem JG, Paty PB, et al. Carcinoid of the rectum risk stratification (CaRRs): a strategy for preoperative outcome assessment. Ann Surg Oncol. 2007;14(5):1735-43.

6. Ushigome H, Fukunaga Y, Nagasaki T, Akiyoshi T, Konishi T, Fujimoto Y, et al. Difficulty of predicting lymph node metastasis on $\mathrm{CT}$ in patients with rectal neuroendocrine tumors. PLoS One. 2019;14(2):e0211675.

7. Takatsu Y, Fukunaga Y, Nagasaki T, Akiyoshi T, Konishi T, Fujimoto Y, et al, Short- and long-term outcomes of laparoscopic total mesenteric excision for neuroendocrine tumors of the rectum. Dis Colon Rectum. 2017:60(3): 284-9.

8. Caplin M, Sundin A, Nillson O, Baum RP, Klose KJ, Kelestimur F, et al. ENETS Consensus Guidelines for the management of patients with digestive neuroendocrine neoplasms: colorectal neuroendocrine neoplasms. Neuroendocrinology. 2012;95(2):88-97.

9. de Mestier L, Brixi H, Gincul R, Ponchon T, Cadiot G. Updating the management of patients with rectal neuroendocrine tumors. Endoscopy. 2013:45(12):1039-46.

10. National Comprehensive Cancer Network. Neuroendocrine and adrenal tumors (Version 1.2019) 2019 [Available from: https://www.ncen.org/ professionals/physician_gls/pdf/neuroendocrine.pdf.

11. Anthony LB, Strosberg JR, Klimstra DS, Maples WJ, O'Dorisio TM, Warner RR, et al. The NANETS consensus guidelines for the diagnosis and management of gastrointestinal neuroendocrine tumors (nets): well-differentiated nets of the distal colon and rectum. Pancreas. 2010;39(6):767-74.

12. Onozato Y, Kakizaki S, lizuka H, Sohara N, Mori M, Itoh H. Endoscopic treatment of rectal carcinoid tumors. Dis Colon Rectum. 2010;53(2):169-76.

13. Bertani E, Ravizza D, Milione M, Massironi S, Grana CM, Zerini D, et al. Neuroendocrine neoplasms of rectum: a management update. Cancer Treat Rev. 2018;66:45-55.

14. Soga J. Carcinoids of the rectum: an evaluation of 1271 reported cases. Surg Today. 1997;27(2):112-9.

15. Konishi T, Watanabe T, Kishimoto J, Kotake K, Muto T, Nagawa H. Prognosis and risk factors of metastasis in colorectal carcinoids: results of a nationwide registry over 15 years. Gut. 2007;56(6):863-8.

16. Matsuhashi N, Takahashi T, Tomita H, Araki H, Ibuka T, Tanaka K, et al. Evaluation of treatment for rectal neuroendocrine tumors sized under 20 $\mathrm{mm}$ in comparison with the WHO 2010 guidelines. Molecular and clinical oncology. 2017;7(3):476-80.

17. Glazer ES, Tseng JF, Al-Refaie W, Solorzano CC, Liu P, Willborn KA, et al. Long-term survival after surgical management of neuroendocrine hepatic metastases. HPB : the official journal of the International Hepato Pancreato Biliary Association. 2010;12(6):427-33.

18. Hashiguchi Y, Muro K, Saito Y, Ito Y, Ajioka Y, Hamaguchi T, et al. Japanese Society for Cancer of the Colon and Rectum (JSCCR) guidelines 2019 for the treatment of colorectal cancer. Int J Clin Oncol. 2019.

\section{Publisher's Note}

Springer Nature remains neutral with regard to jurisdictional claims in published maps and institutional affiliations. 